\title{
Experiment on the Self-Aggregation of Amphiphiles
}

\author{
Elena von Hoff ${ }^{1}$, Silan Toy ${ }^{1}$, Thomas Waitz ${ }^{1}$, Ingo Mey ${ }^{2, *}$ \\ ${ }^{1}$ Institute of Inorganic Chemistry, Department of Chemistry Education, Georg-August-University, Göttingen, Germany \\ ${ }^{2}$ Institute of Organic and Biomolecular Chemistry, Georg-August-University, Göttingen, Germany \\ *Corresponding author: imey@gwdg.de
}

Received January 16, 2019; Revised March 14, 2019; Accepted April 10, 2019

\begin{abstract}
Formation of supramolecular aggregates such as micelles and vesicles is governed by hydrophobic and hydrophilic interaction as well as the geometric shapes of the aggregating molecules. Here we present an experiment for upper secondary chemistry class to show how structural properties of molecules such as fatty acids govern the shapes of aggregates. For fatty acids, the $\mathrm{pH}$ value controls whether micelles or vesicles are formed. Using a size exclusion column and the possibility to encase fluorescing dye molecules in vesicles, the properties of micelles and vesicles are explored and compared to typical micelle building surfactants from dish detergent and the vesicle building lecithin.
\end{abstract}

Keywords: self-aggregation, size exclusion column, surfactants, lipids, vesicles, micelles

Cite This Article: Elena von Hoff, Silan Toy, Thomas Waitz, and Ingo Mey, "Experiment on the Self-Aggregation of Amphiphiles." World Journal of Chemical Education, vol. 7, no. 2 (2019): 185-188. doi: $10.12691 /$ wjce-7-2-19.

\section{Introduction}

Self-aggregation plays an important role in chemical and biological processes, typically governed by simple chemical driving forces, e.g. hydrophilic and/or hydrophobic interactions, hydrogen bonds and pi-pi stacking [1]. Aggregates determine phenomena such as structural conformation in proteins and DNA, reduction of surface tension due to surfactants and the formation of biologically relevant structures such as micelles, important for fat storage within cells, or vesicles, playing an essential role for substance transport in living organisms. Especially supramolecular aggregates such as micelles and vesicles are consistently important in research for e.g. reconstitution processes of proteins in biochemistry [2] or, in the case of vesicles, are subject of current research on biological transport phenomena [3]. Here we present experiments on the formation and characteristics of micelles and vesicles as supramolecular aggregates, following the work of Bianco et al [4], but adjusting it to complement chemistry and biology teaching in school. The experimental setup is based on well-known structural assemblies of fatty acids [1] and a very simple setup for size exclusion chromatography.

While the formation of micelles is typically referred to regarding washing processes using surfactants from dish detergents or soap, vesicles are mentioned with regard to the synaptic transport of neurotransmitters [5] as consisting of phospholipids and to some extent sphingolipids. However, the common basic principles behind these aggregates as well as how molecular geometry and chemical interactions drive the geometry of molecular aggregates are rarely discussed.
A1

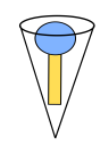

A2

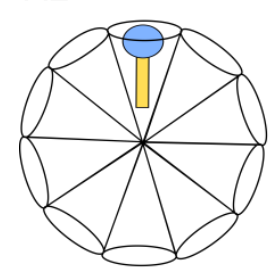

A3

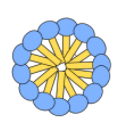

B1

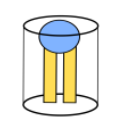

B2

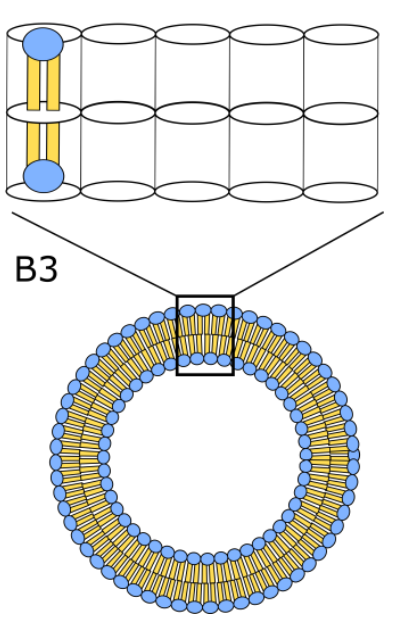

Figure 1. A1) conical shape of surfactant molecules consisting of a hydrophilic headgroup (blue) and a hydrophobic alkyl chain (yellow). A2, A3) supramolecular aggregates of the surfactant in A1), showing how molecular geometry leads to formation of micelles. B1) cylindrical shape of typical lipid molecules, consisting of a hydrophilic headgroup and two hydrophobic alkyl chains. B2, B3) supramolecular aggregates of these molecules tend to form bilayer structures such as vesicles

While the general formation of micelles and vesicles is driven by the amphiphilic nature of surfactants and lipids, which type of aggregate is formed depends mostly on the geometric shape of the molecules [1]. Typically, surfactants in dish detergents have a highly charged or at 
least a bulky hydrophilic headgroup and only one alkyl chain, leading to a conical shape as shown in Figure 1 (A1). Supramolecular aggregates of these molecules lead to micelles as schematically shown in A2 and A3. Lipids, such as phospholipids from e.g. lecithin, a main component of eukaryotic cellular membranes $[6,7,8]$, typically have a larger headgroup but also 2 alkyl chains, which resembles a more cylindrical structure as depicted B1. Aggregation of these molecules leads to bilayer structures such as vesicles, as presented in B2 and B3. Contrary to micelles, where the inside of the sphere consists of hydrophobic lipid chains, vesicles are hollow spheres encapsulating a hydrophilic compartment as the walls are built of a lipid-bilayer structure.

\section{Experiments}

\subsection{Preparation and Visualization of Supra-molecular Aggregates Using Fluorescent Dye}

\section{Chemicals and Equipment:}

Water, linolenic (85\% TCI Deutschland GmbH, Germany), palmitic $(97,0 \%$, TCI Deutschland $\mathrm{GmbH}$, Germany) or oleic acid (85\%, TCI Deutschland GmbH, Germany), lecithin (egg) $(70 \%$, TCI Deutschland GmbH. Germany), dish detergent, pyranine (Sigma Aldrich, Germany), sodium hydroxide solution $(0.1 \mathrm{M})$, hydrochloric acid solution $(0.1 \mathrm{M})$, size exclusion column (sephadex G25, VWR Germany), spatula, Pasteur pipettes, support stand with clamps, 11 beakers $(10 \times 50 \mathrm{~mL}, 1 \times 500 \mathrm{~mL})$, pH-meter, UV light source, ultrasonicator, magnetic stirrer.

Experimental:

While stirring, 4 spatula tips of pyranine are solved in $500 \mathrm{~mL}$ water. The $\mathrm{pH}$ of the solution is measured and if necessary set to around $\mathrm{pH}=8$ using either hydrochloric acid or sodium hydroxide solution. $40 \mathrm{~mL}$ of the solution is given into five $50 \mathrm{~mL}$ beakers respectively and the following is added: (A) a spatula tip of dish detergent, B2) a spatula tip of lecithin, (C) a spatula tip of fatty acid, (D) a spatula tip of fatty acid and sodium hydroxide solution, setting the $\mathrm{pH}$ to 11 . The content of the fifth beaker (E) remains the same. All five solutions are ultrasonicated for at least 5 minutes (10 if a non-laboratory ultrasonicator is used) and left to rest for another 5 minutes.

For the size exclusion chromatography, all following steps are done under UV light exposure to enhance the visibility of the pyranine. It is possible to use one column for all solutions (rinsing of the column required), using different columns, however, allows a comparison of results. For each solution a column (as seen in Figure 2) is opened until the top of the column is nearly dry.

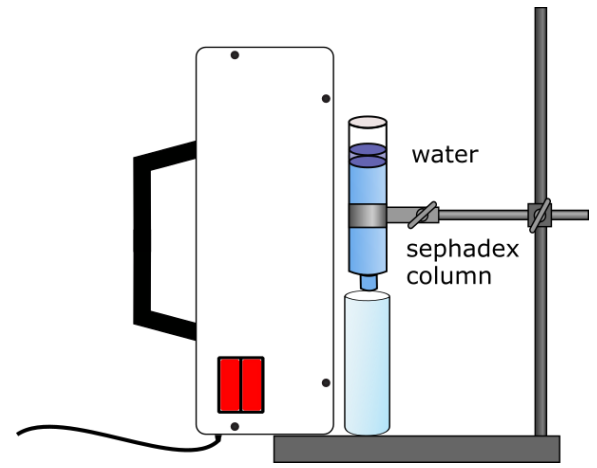

Figure 2. Experimental setup for the size exclusion chromatography

Two droplets of the respective solution are gently poured on top of the column material. After the solution has sunk into the material, a few droplets of pure or distilled water are added until a colored band at the top of the column is formed. Subsequently the column is gently filled almost to the rim with water. The solution dripping from the column is collected until the observed fluorescence is approximately at the middle of the column. To stop the process, the column is again closed at the bottom.
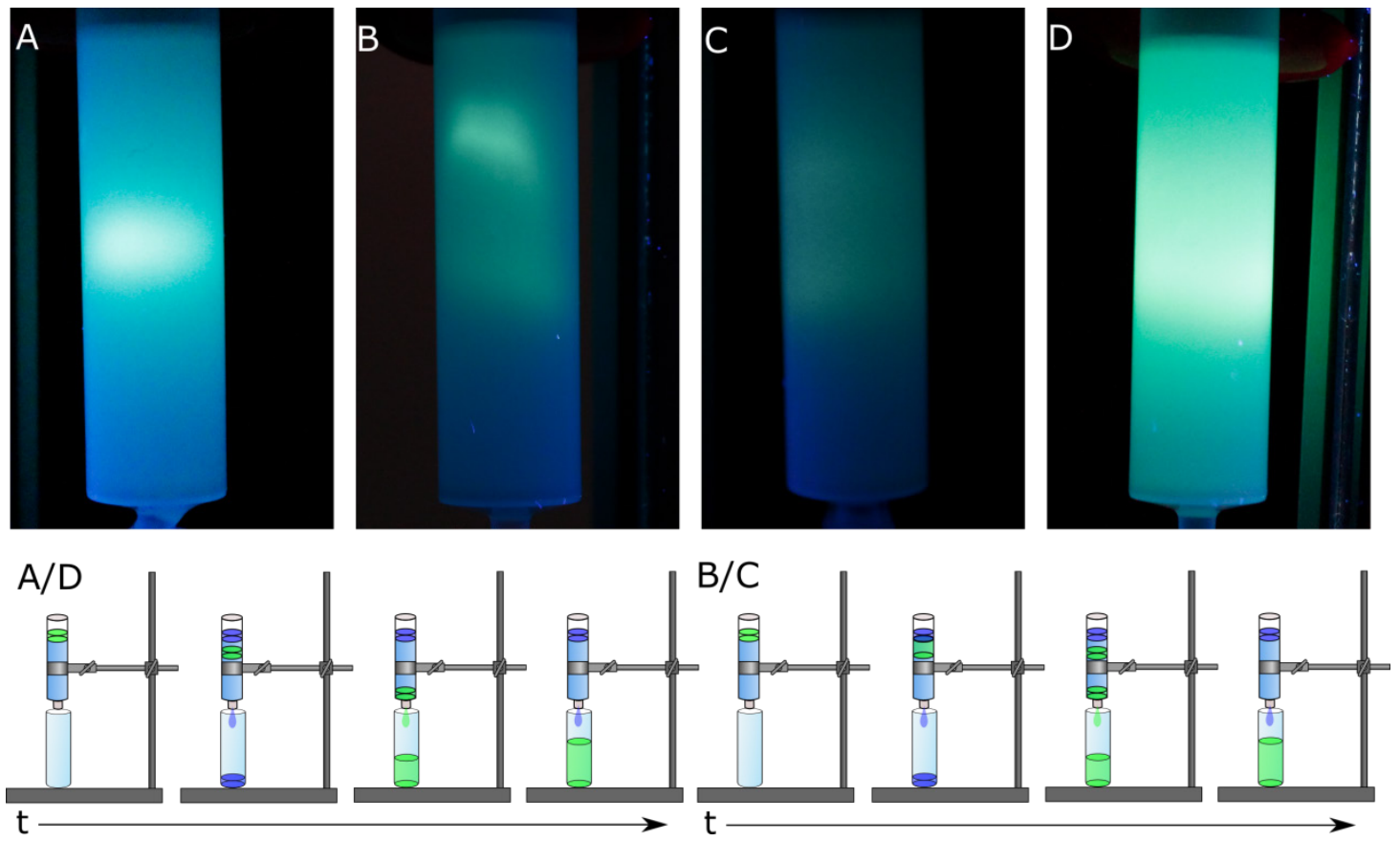

Figure 3. Size exclusion columns (Sephadex 25) of A) dish detergent + pyranine solution, B) lecithin + pyranine solution, C) oleic acid + pyranine solution at $\mathrm{pH}=8$ and $\mathrm{D}$ ) oleic acid + pyranine solution at $\mathrm{pH}=11$ are shown. Pyranine is excited via UV light source (254nm and $356 \mathrm{~nm})$ 


\section{Observation:}

Adding pyranine to water leads to a transparent yellow solution, fluorescing yellowish-green under UV light. After ultrasonication suspensions can be observed in beakers A through $\mathrm{D}$, while the solution in beaker $\mathrm{E}$ stays clear (see Figure 4).

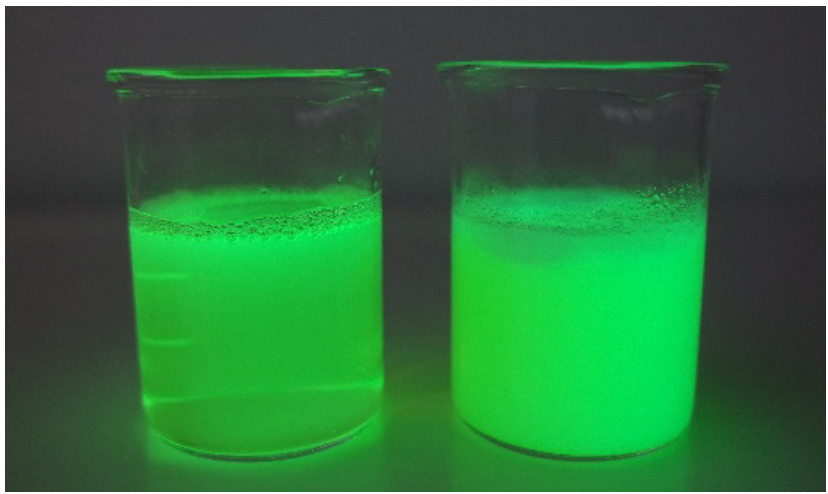

Figure 4. Solutions containing water and pyranine (left) and water, pyranine and lecithin (right), after sonication and under UV light exposure

Within the columns the formation of fluorescing bands can be observed under UV light exposure for the respective solutions: (A) one band, (B) two bands, (C) two bands, (D) one band, (E) one ban (Figure 3).
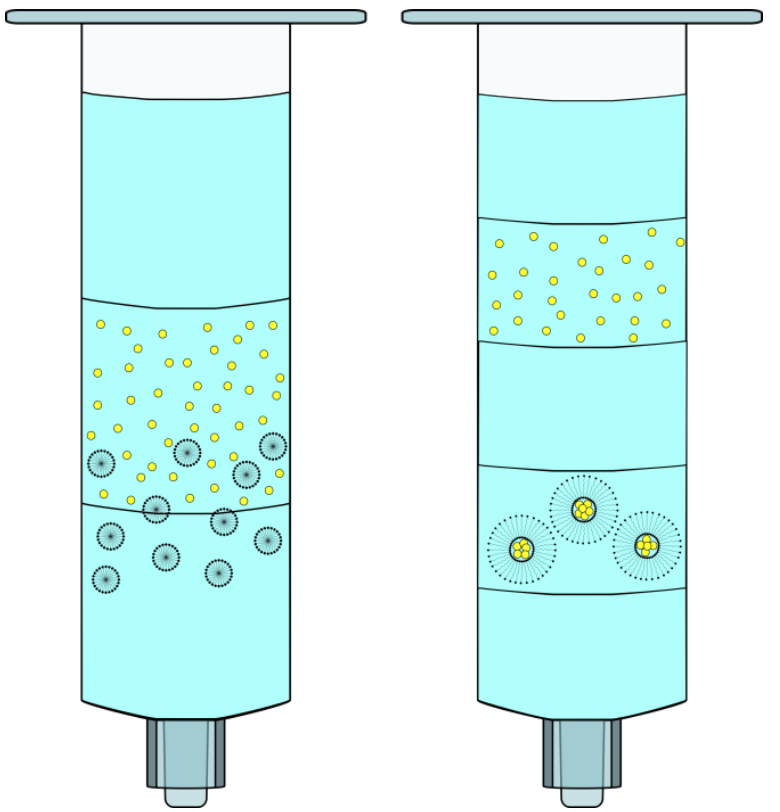

Figure 5. Schematic of the columns' content on a molecular level. Left: only one band can be observed for solutions A and D. The micelles are not able to carry fluorescent dye molecules (however, Tyndall-effect showed, that micelles and dye were not entirely separated by the size exclusion column). Right: vesicular aggregates encase dye molecules, leading to two fluorescing bands.

\section{Explanation:}

When dish detergent, lecithin and fatty acids are dissolved in the colored solution, aggregates with sizes in the nanometer range form based on the amphiphilic nature of the molecules. During the column chromatography the different components of the solution are separated mainly based on their molecular sizes. For the first column including the dish detergent, only one fluorescing band can be observed. Dish detergent aggregates to micelles when solved in water, which cannot be observed on the column. Therefore, only one band formed by the pyranine can be observed, with the fluorescence a characteristic of the pyranine. Lecithin, as a lipid with two hydrophobic chains, is too bulky to form micelles and instead aggregates to vesicles, as previously shown in Figure 1B. During the vesicle formation pyranine molecules are encased inside the hollow spheres, leading to two colored bands on the column - the first for the pyranine encased in the significantly bulkier vesicles, the second for the free pyranine (see Figure 5). Depending on the $\mathrm{pH}$ of the fatty acid suspensions, both one $(\mathrm{pH}=11)$ and two $(\mathrm{pH}=8)$ fluorescing bands can be observed. Apparently the $\mathrm{pH}$-value has an influence on the aggregation of the molecules, leading to both micelles and vesicles respectively. The fifth solution without any additives shows only one band, merely separating the pyranine from the water.

\section{Scientific Comments}

The self-aggregation of amphiphilic molecules is driven by the interaction of the hydrophilic headgroups with each other and the water, as well as the interaction of hydrophobic residues, often referred to as the hydrophobic effect [1]. Hereby, the geometrical shape of the molecule, as shown in Figure 1, determines the structure of the supramolecular aggregate.

Interestingly for the case of fatty acids the supramolecular aggregate depends on the $\mathrm{pH}$ value of the solution $[4,9,10]$. One would expect full deprotonation above $\mathrm{pH}$ values of 4 , as typically the $\mathrm{pKs}$ for carboxylic acids is around 4 [9]. However, polyanionic surfaces as found in the fatty acid aggregates have strong electrostatic effects, depending on the ion strength and charge density. $\mathrm{pH}$ values near the surface can therefore differ as much as 3 units from the $\mathrm{pH}$ value of the bulk solution, resulting in vesicle formation in the $\mathrm{pH}$ range of 7-10 for partially deprotonated fatty acids and above $\mathrm{pH}=10$ to full deprotonation and thus micelle formation. Using solutions with $\mathrm{pH}<7$ leads to protonation and formation of oil droplets [9]. Since at high $\mathrm{pH}$ values the acidic groups are fully deprotonated, the strong headgroup charge leads to a cone like geometry (Figure 6D) which is typical for the formation of micellar structures also found in surfactants (Figure 6A) [9]. Lowering the $\mathrm{pH}$ to around 8 leads to partial protonation, allowing for dimerization of the fatty acids [9]. These dimers form larger hydrophilic headgroups, composed of two carboxylic groups, connected via a hydrogen bond, as depicted in Figure 6C. Based on the two alkyl chains connected to the dimeric headgroup, the resulting structure resembles phosphocholine lipids found in lecithin (Figure 6B), which are well known to form vesicles due to their cylindrical geometry. Accordingly, two fluorescent bands can be observed in the size exclusion column, the upper one containing dye-carrying vesicles.

For saturated, mono- and poly-unsaturated fatty acids (linolenic, palmitic and oleic acid) the observed behavior was consistent, therefore the hydrophobic interactions drives aggregation but does not determine whether vesicles or micelles are formed. 
Vesicles formed by lipids are typically very stable to changes of the surrounding media [11] while fatty acids can change their aggregation state and therefore react to changes in the bulk solution. In comparison to phosphocholine lipid molecules, the stability of fatty acid vesicles is significantly lower. In phosphocholine lipids, glycerin serves as the backbone connecting two alkyl chains with a hydrophilic headgroup. The fatty acid dimer, however, is only stabilized via one hydrogen bond. While supramolecular aggregates are stable against $\mathrm{pH}$ changes, sonication exerts enough energy to break the hydrogen bond, allowing for faster deprotonation or protonation and subsequent reorganization. Without sonification these processes would also work but might take much more time.

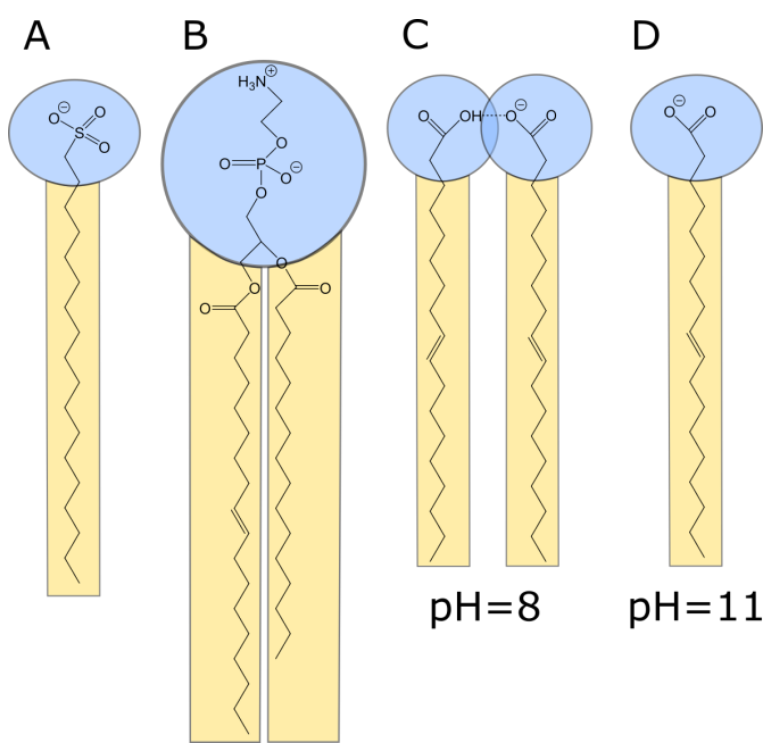

Figure 6. A) typical sulfonate surfactant, due to the conical shape micelles are formed in solution. B) typical structure of a phosphocholine lipid found in lecithin, due to two alkyl chains the geometry is cylinderlike. C) at $\mathrm{pH}=8$ oleic acid is partially deprotonated and aggregates of two molecules are form, resulting in a lipid-like structure. These dimers are also cylindrical in geometric shape, therefore vesicles are formed, while at $\mathrm{D}) \mathrm{pH}=11$ all fatty acids are deprotonated, resulting in a conical, micelle-forming shape

\section{Educational Perspectives}

The presented experiment offers an illustrative approach to demonstrate how molecular geometry and chemical interactions drive the geometry of molecular aggregates. For this purpose, the method of size exclusion chromatography, an important research tool e.g. in biochemistry, is used. As a low cost and quick alternative for chemistry class, commercially available plastic syringes were chosen as columns.

With regards to nature it can be shown why lipids, forming cylindrical structures, are better suited as components for natural barriers such as cell membranes than surfactants. For cellular membranes the bilayer structure is integral to divide two hydrophilic compartments, cell interior and exterior, allowing to generate chemical and electrical gradients which are the foundation for living organisms. Equivalent to what is found for vesicles during the experiment - entrapping pyranine and transporting it, separated from free pyranine, across the column - cells encase molecules such as neurotransmitters in vesicles for substance transportation within the cells and across cellular membranes. The substances are then released at the targeted destination, avoiding unintended interaction of the molecules.

For the interpretation of the findings, knowledge of structure-property (s-p) as well as donor-acceptor (d-a) concepts and their interplay is crucial. The formation of the supramolecular aggregates is based on the amphiphilic nature of the underlying molecules as well as their geometrical shapes (s-p). Changing the $\mathrm{pH}$ value leads to (de)protonation (d-a) which in return changes the geometric structure of the molecules (dimer formation), affecting their properties and therefore their aggregation behavior (s-p).

\section{Acknowledgements}

This work was supported by the Deutsche Forschungsgemeinschaft (SFB 803, project Ö01). We thank Jutta Gerber-Nolte and Markus Schön for technical and scientific assistance.

\section{References}

[1] D. Marsh, Handbook of lipid bilayers, 2nd ed. Boca Raton, FL: CRC Press, Taylor \& Francis Group, 2013.

[2] J.-L. Rigaud und D. Lévy, "Reconstitution of Membrane Proteins into Liposomes", in Methods in Enzymology, 372, 65-86, 2003.

[3] M. K. Domanska, V. Kiessling, und L. K. Tamm, "Docking and fast fusion of synaptobrevin vesicles depends on the lipid compositions of the vesicle and the acceptor SNARE complex-containing target membrane", Biophys. J., 99 (9), 2936-2946, 2010.

[4] C. D. Bianco, D. Torino, und S. S. Mansy, „Vesicle Stability and Dynamics: An Undergraduate Biochemistry Laboratory“, J. Chem. Educ., 91 (8), 1228-1231, Aug. 2014.

[5] Y. A. Chen und R. H. Scheller, "SNARE-mediated membrane fusion", Nat. Rev. Mol. Cell Biol., 2 (2), 98-106, Feb. 2001.

[6] W. C. Breckenridge, G. Gombos, und I. G. Morgan, "The lipid composition of adult rat brain synaptosomal plasma membranes", Biochim. Biophys. Acta BBA - Biomembr., 266 (3), 695-707, 1972.

[7] W. C. Breckenridge, I. G. Morgan, J. P. Zanetta, und G. Vincendon, "Adult rat brain synaptic vesicles II. Lipid composition", Biochim. Biophys. Acta BBA - Gen. Subj., 320 (3), 681-686, 1973.

[8] A. A. Spector und M. A. Yorek, "Membrane lipid composition and cellular function”, J Lipid Res, 26 (9), 21, 1985.

[9] T. H. Haines, „Anionic lipid headgroups as a proton-conducting pathway along the surface of membranes: a hypothesis“, Proc. Natl. Acad. Sci., 80 (1), 160-164, Jan. 1983.

[10] J. M. Gebicki und M. Hicks, "Ufasomes are Stable Particles surrounded by Unsaturated Fatty Acid Membranes", Nature, 243 (5404), 232-234, Mai 1973.

[11] P. L. Luisi, "Are Micelles and Vesicles Chemical Equilibrium Systems?”, J Chem Educ, 78 (3), 380, 2001. 